\title{
Genetic Predictors of Poor Prognosis in Portuguese Patients with Juvenile Idiopathic Arthritis: Data from Reuma.pt
}

\author{
Ana Filipa Mourão, ${ }^{1,2,3}$ Maria José Santos, ${ }^{1,4}$ Sílvia Mendonça, ${ }^{5}$ Filipa Oliveira-Ramos, ${ }^{6}$ \\ Manuel Salgado, ${ }^{7}$ Paula Estanqueiro, ${ }^{7}$ José Melo-Gomes, ${ }^{5,8}$ Fernando Martins, ${ }^{5}$ \\ Ana Lopes, ${ }^{1}$ Bruno Filipe Bettencourt, ${ }^{9,10,11}$ Jácome Bruges-Armas, ${ }^{9,10,11}$ José Costa, ${ }^{12}$ \\ Carolina Furtado, ${ }^{13}$ Ricardo Figueira, ${ }^{14}$ Iva Brito, ${ }^{15,16}$ Jaime Branco, ${ }^{2,3}$ \\ João Eurico Fonseca, ${ }^{1,6}$ and Helena Canhão ${ }^{1,6}$ \\ ${ }^{1}$ Rheumatology Research Unit, Instituto de Medicina Molecular, 1649-028 Lisbon, Portugal \\ ${ }^{2}$ Rheumatology Department, Hospital Egas Moniz, Centro Hospitalar Lisboa Ocidental, 1349-019 Lisbon, Portugal \\ ${ }^{3}$ CEDOC, Faculdade de Ciências Medicas da Universidade Nova de Lisboa, 1150-190 Lisbon, Portugal \\ ${ }^{4}$ Rheumatology Department, Hospital Garcia de Orta, 2801-951 Almada, Portugal \\ ${ }^{5}$ Portuguese Society of Rheumatology, 1800-033 Lisbon, Portugal \\ ${ }^{6}$ Rheumatology Department, Lisbon Academic Medical Center, 1649-028 Lisbon, Portugal \\ ${ }^{7}$ Pediatrics Department, Centro Universitário Hospitalar de Coimbra, 3041-801 Coimbra, Portugal \\ ${ }^{8}$ Instituto Português de Reumatologia, 1601-901 Lisbon, Portugal \\ ${ }^{9}$ Institute for Molecular and Cell Biology (IBMC), University of Porto, 4150-180 Porto, Portugal \\ ${ }^{10}$ Hospital de Santo Espírito da Ilha Terceira, SEEBMO, Angra do Heroísmo, 9700-049 Açores, Portugal \\ ${ }^{11}$ Instituto de Investigação e Inovação em Saúde, Universidade do Porto, 4150-180 Porto, Portugal \\ ${ }^{12}$ Rheumatology Department, Hospital Conde de Bertiandos, ULSAM, 4990-041 Ponte de Lima, Portugal \\ ${ }^{13}$ Rheumatology Department, Hospital do Divino Espírito Santo, São Miguel, 9500-370 Açores, Portugal \\ ${ }^{14}$ Rheumatology Department, Hospital Dr. Nélio Mendonça, Funchal, 9004-514 Madeira, Portugal \\ ${ }^{15}$ Rheumatology Department, Hospital de São João, 4200-319 Porto, Portugal \\ ${ }^{16}$ Faculdade de Medicina da Universidade do Porto, 4200-450 Porto, Portugal
}

Correspondence should be addressed to Ana Filipa Mourão; afilipamourao@gmail.com

Received 5 May 2015; Accepted 14 May 2015

Academic Editor: Carlo Perricone

Copyright (C) 2015 Ana Filipa Mourão et al. This is an open access article distributed under the Creative Commons Attribution License, which permits unrestricted use, distribution, and reproduction in any medium, provided the original work is properly cited.

Introduction. This study aimed to assess the genetic determinants of poor outcome in Portuguese patients with juvenile idiopathic arthritis (JIA). Methods. Our study was conducted in Reuma.pt, the Rheumatic Diseases Portuguese Register, which includes patients with JIA. We collected prospectively patient and disease characteristics and a blood sample for DNA analysis. Poor prognosis was defined as $\mathrm{CHAQ} / \mathrm{HAQ}>0.75$ at the last visit and/or the treatment with biological therapy. A selected panel of single nucleotide polymorphisms (SNPs) associated with susceptibility was studied to verify if there was association with poor prognosis. Results. Of the 812 patients with JIA registered in Reuma.pt, 267 had a blood sample and registered information used to define "poor prognosis." In univariate analysis, we found significant associations with poor prognosis for allele A of TNFA1P3/20 rs6920220, allele $\mathrm{G}$ of TRAF1/C5 rs3761847, and allele G of PTPN2 rs7234029. In multivariate models, the associations with TRAF1/C5 (1.96 [1.17-3.3]) remained significant at the 5\% level, while TNFA1P3/20 and PTPN2 were no longer significant. Nevertheless, none of associations found was significant after the Bonferroni correction was applied. Conclusion. Our study does not confirm the association between a panel of selected SNP and poor prognosis in Portuguese patients with JIA. 


\section{Introduction}

Juvenile idiopathic arthritis (JIA) is the most common childhood rheumatic disease [1]. Despite significant improvements in the management of children with JIA, the likelihood of long-term persistent disease activity remains high [2]. Published evidence demonstrates that clinical subtype, disease activity and duration, and response to treatment all influence the prognosis $[3,4]$. In addition, diagnostic delay, severity and extension of arthritis at onset, symmetric disease, early hip or wrist involvement, involvement of cervical spine, the presence of rheumatoid factor (RF) or anticyclic citrullinated peptide, early age at onset, female gender, and family history of rheumatic disease were the best predictors of a poor outcome [3, 5-11]. However, in most studies of prognostic predictors in JIA, the authors are unanimous in concluding that there is considerable variability in results, making it harder to draw consistent conclusions [8].

Identifying earlier JIA worse prognosis cases is crucial to start appropriate treatment and to correctly inform patients and their parents. Much effort has already been done to elucidate prognosis predictors. Besides clinical factors, identification of genetic predictors of poor prognosis would be a significant contribution to the development of optimal treatment strategies for JIA.

Studies that evaluate nonclinical predictors, such as genetic or immunological parameters, hardly exist. Most of the genetic research aimed to identify variants that affect the risk of developing JIA or pathways modulating drug response in this disease. On the contrary, the goal of this study was to assess the genetic determinants of poor outcome in Portuguese patients with JIA. Our secondary objective was to find potential clinical predictors of poor prognosis.

\section{Methods}

2.1. Patient Population. Our study was conducted based on Reuma.pt, the Rheumatic Diseases Portuguese Register, which includes JIA patients treated with synthetic and biological Disease Modifying Antirheumatic Drugs (DMARDs) since June 2001. Patients registered up to December 2013 were included. The parent's consent and patient's assent (as appropriate) were obtained according to the declaration of Helsinki. The study was approved by local Ethics Committee. All patients fulfilled the ILAR criteria for the classification of JIA [12]. This study did not have any interference with patients' standard of care.

We analyzed the patients registered in Reuma.pt with the diagnosis of JIA, who had collected a blood sample for DNA analysis. The following data were collected at the time of the last visit to rheumatology clinics: gender, age, JIA subtype, disease duration, time until diagnosis (time since the beginning of the symptoms until the diagnosis of JIA), extraarticular manifestations of the disease, duration of therapy with DMARDs, corticosteroids and biological therapies, Childhood Health Assessment Questionnaire (CHAQ)/ Health Assessment Questionnaire (HAQ) [13], patient's/ parent's pain visual analogue scale (VAS), patient's/parent's disease global activity VAS, and physician's global disease activity VAS.

One of the barriers found in prognostic studies of JIA is that there is no universal definition of "poor prognosis." We have chosen to integrate in our definition of "poor prognosis" two variables: one instrument that combines disease activity and damage (CHAQ), dichotomized in accordance with other studies [14-19], using 0.75 as the cut-off point, combined with "the need for biological therapy," as a surrogate marker of disease severity and higher likelihood of a worse outcome. We have classified as patients in "need for biological therapy" all patients that were ever treated with biological agents for more than 3 months, due to articular or extraarticular manifestations of the disease. Thus, for the purpose of this study a patient was classified as having poor prognosis if CHAQ >0.75 and/or if the patient was ever treated with biological therapy for more than 3 months.

Genetic single nucleotide polymorphisms (SNPs) were studied to verify if there was any association with poor prognosis.

2.2. Genetic Analysis. The choice of SNP variants was based on information from previous studies of susceptibility and prognosis factors in JIA and included the following 32 SNPs of genes with a known function in the immune system: PTPN22 rs2476601, PTPRC rs10919563, TNFAIP3/A20 rs10499194, TNFAIP3/A20 rs6920220, TRAF1/C5 rs3761847, ANGPT1 rs1010824, ANGPT1 rs7151781, AFF3 rs1160542, AFF3 rs10865035, CTLA4 rs3087243, ERAP1/ARTS1 rs30187, IL1 rs6712572, IL1 rs2071374, IL1 rs1688075, IL10-1080GA rs1800896, IL10-819CT rs1800871, IL1R rs12712122, IL23R rs11209026, IL2-IL21 rs6822844, IL2RA/CD25 rs2104286, MIF-173CG rs755622, PTPN2 rs1893217, PTPN2 rs7234029, SLC26A2 rs1541915, STAT4 rs3821236, STAT4 rs7574865, TNF-238 rs361525, TNF-308 rs1800629, VTCN1 rs10923223, VTCN1 rs12046117, WISP3 rs2280153, and EYA4 rs17301249.

All samples were genotyped using Taqman SNP genotyping assays (Applied Biosystems, Foster City, USA) performed as described in the manufacturers' protocol. Genotyping reactions were carried out with an $\mathrm{ABI} 7500$-fast thermocycler. The allele call was obtained by the AB software v2.0.5, by the analysis of allelic discrimination plots. SNPs with deviation from Hardy-Weinberg equilibrium $(P<0.05)$ or minor allele frequency (MAF) $<1 \%$ were excluded from further analysis.

2.3. Statistical Analysis. We used the additive model to study the association between SNPs and poor prognosis, where homozygotes for the major allele were classified as zero, heterozygotes as 1, and homozygotes for the minor allele as 2. We report crude odds ratio (OR) based on a univariate logistic regression and adjusted $\mathrm{OR}$ from a multivariate model including significant clinical predictors. The following clinical variables were characterized: gender, disease category (classified into five groups including polyarticular JIA (RF negative, RF positive, and extended oligoarticular), persistent oligoarticular JIA, systemic arthritis, enthesitis-related arthritis (ERA), and psoriatic arthritis), time until diagnosis 
TABLE 1: Distribution of the clinical characteristics of patients with and without poor prognosis.

\begin{tabular}{|c|c|c|c|c|}
\hline Variable & Total & Patients with poor prognosis & Patients without poor prognosis & $P$ value \\
\hline Number & 267 & 85 & 182 & \\
\hline Female gender $n(\%)$ & $171(64)$ & $60(22.59)$ & $111(41.6)$ & 0.166 \\
\hline \multicolumn{5}{|l|}{ JIA categories (\%): } \\
\hline Polyarticular RF negative & $48(18)$ & $19(39.6)$ & $29(60.4)$ & \multirow{7}{*}{$<0.001$} \\
\hline Polyarticular RF positive & $25(9.4)$ & $19(76)$ & $6(24)$ & \\
\hline Extended oligoarticular & $43(16.1)$ & $17(39.5)$ & $26(60.5)$ & \\
\hline Persistent oligoarticular & $89(33.3)$ & $7(7.9)$ & $82(92.1)$ & \\
\hline Systemic & $22(8.2)$ & $11(50)$ & $11(50)$ & \\
\hline Enthesitis-related arthritis & $28(10.5)$ & $8(28.6)$ & $20(71.4)$ & \\
\hline Psoriatic arthritis & $12(4.5)$ & $4(33.3)$ & $8(66.7)$ & \\
\hline Age at disease onset (median (IQR)) & $5.3(2.2-9.7)$ & $6.6(3.1-11.6)$ & $4.8(2.1-8.8)$ & 0.056 \\
\hline Age at diagnosis (median (IQR)) & $6.6(2.8-11.6)$ & $8.7(3.4-13.7)$ & $5.7(2.5-10.6)$ & 0.013 \\
\hline Time until diagnosis (median (IQR)) & $0.33(1.14-1.00)$ & $0.50(0.17-1.0)$ & $0.26(0.14-0.88)$ & 0.130 \\
\hline Age at last visit (median (IQR)) & $14.3(8.9-18.3)$ & $16.9(13.1-24.1)$ & $12.7(6.9-13.3)$ & $<0.001$ \\
\hline Disease duration (median (IQR)) & $6.4(3.1-12.0)$ & $10.4(5.2-16.0)$ & $4.9(2.3-10.4)$ & $<0.001$ \\
\hline CHAQ/HAQ (median (IQR)) & $0(0-0.25)$ & $0.25(0-1)$ & $0(0-0.13)$ & $<0.001$ \\
\hline Patient's/parent's VAS (median (IQR)) & $5(0-30)$ & $10(0-50)$ & $0(0-30)$ & 0.017 \\
\hline Physician VAS (median (IQR)) & $0(0-20)$ & $10(0-35)$ & $0(0-11.3)$ & $<0.001$ \\
\hline Extra-articular manifestations & 98 & 41 & 57 & 0.011 \\
\hline Duration of DMARD use (median (IQR)) & $2.37(0-5.8)$ & $5.46(3.02-9.54)$ & $1.43(0-4.02)$ & $<0.001$ \\
\hline Corticosteroid use $(\mathrm{Y} / \mathrm{N})$ & 124 & 52 & 68 & $<0.001$ \\
\hline
\end{tabular}

F: female; M: male; JIA: juvenile idiopathic arthritis; RF: rheumatoid factor; IQR: interquartile range; CHAQ: Childhood Health Assessment Questionnaire; HAQ: Health Assessment Questionnaire; SD: standard deviation; VAS: visual analogue scale; DMARD: Disease Modifying Antirheumatic Drug; Y: yes; N: no. $P$ values $<0.05$ were considered statistically significant. Note: $P$ values are from Pearson's chi-squared or Mann-Whitney tests, as appropriate.

(years), age at disease onset, disease duration (years), duration of DMARD treatment (years), corticosteroid treatment (ever or never), patient's/parent's disease global activity VAS, physician's global disease activity VAS, and extra-articular manifestations (yes or no). Continuous variables were modelled as linear. All clinical variables crudely associated with poor prognosis $(P<0.20)$ were included in a multivariate model. Then backward selection was applied to retain the clinical variables most associated with the outcome, using a significance level of 5\%. Due to small sample size for most of the disease categories we carried out the analysis using all JIA categories combined. The stratified analysis was only possible for the polyarticular categories (polyarticular RF positive, polyarticular RF negative, and extended oligoarticular JIA) with 116 patients.

There was missing data for some of the variables, as follows: age at disease onset (1.5\%), age at diagnosis (1.9\%), patient's/parent's VAS (4.9\%), and physician VAS (9.7\%).

Statistical significance was considered at the $5 \%$ level. After Bonferroni correction for the 32 SNPs analyzed, results were considered significant for $P<0.0016$.

Statistical analysis was made in R version 2.15 .3 [20].

\section{Results}

Twenty-one centers and 77 rheumatologists and pediatricians contributed with data to Reuma.pt. Of the 812 patients with JIA registered in Reuma.pt (mean age $19.9 \pm 11.3$ years old,
$65 \%$ females, and mean age at JIA onset $6.9 \pm 4.7$ years old), 291 had a blood sample to perform the genetic analysis and, from those, 267 had registered information about CHAQ/HAQ and/or the need for biological therapy used to define "poor prognosis." Of the 267 patients included, 85 had a poor prognosis, according to the definition: $\mathrm{CHAQ} / \mathrm{HAQ}$ $>0.75$ and/or the treatment with biological therapy for more than 3 months. Nineteen patients had a CHAQ/HAQ $>0.75$ at the last appointment, 58 were treated with biological therapy, and 8 fulfilled both criteria.

Table 1 shows the distribution of the clinical characteristics of patients with and without poor prognosis.

3.1. Clinical Predictors of Poor Prognosis. Almost all the clinical variables, except gender, age at disease onset, and delay in diagnosis, were significantly different between the group of JIA patients with poor prognosis and the group who did not had poor prognosis (Table 1).

Clinical variables significantly associated with poor prognosis and included in the multivariate models were DMARD treatment (OR 1.17 [95\% confidence interval 1.07-1.27]), higher physician VAS (1.03 [1.01-1.04]), and disease category. In particular, the persistent oligoarticular category had a much lower chance of worse prognosis (0.09 [0.04-0.22]) compared to the polyarticular category; ERA (0.44 [0.18$1.09])$, systemic arthritis (1.11 [0.45-2.76]), and psoriatic arthritis $(0.55$ [0.16-1.94]) categories were not significantly different to the polyarticular group of JIA. 
TABLE 2: Crude and adjusted odds ratio for the association between single nucleotide polymorphisms and poor prognosis.

\begin{tabular}{|c|c|c|c|c|c|}
\hline & \multirow{2}{*}{ Minor allele } & \multicolumn{2}{|c|}{ Crude } & \multicolumn{2}{|c|}{ Adjusted $^{\dagger}$} \\
\hline & & OR (95\% CI) & $P$ value & OR $(95 \% \mathrm{CI})$ & $P$ value \\
\hline \multicolumn{6}{|l|}{ All categories } \\
\hline TNFA1P3/20 rs6920220 A/G & A & $1.53(1.01-2.33)$ & 0.0436 & $1.67(0.98-2.83)$ & 0.0579 \\
\hline TRAF1/C5 rs3761847 A/G & G & $1.49(1.00-2.21)$ & 0.0491 & $1.96(1.17-3.3)$ & 0.0110 \\
\hline PTPN2 rs7234029 A/G & G & $1.86(1.17-2.95)$ & 0.0085 & $1.75(0.99-3.1)$ & 0.0540 \\
\hline \multicolumn{6}{|l|}{ Polyarticular categories } \\
\hline CTLA4 rs3087243 A/G & A & $1.98(1.14-3.45)$ & 0.0153 & $2.9(1.39-6.08)$ & 0.0047 \\
\hline PTPN2 rs7234029 A/G & G & $3.08(1.53-6.19)$ & 0.0016 & $3.3(1.48-7.37)$ & 0.0035 \\
\hline
\end{tabular}

${ }^{\dagger}$ Clinical covariates included disease category, DMARD treatment, and physician VAS. Disease category was omitted from the model for the polyarticular categories of JIA.

OR: odds ratio; SNPs: single nucleotide polymorphisms. $P$ values $<0.05$ were considered statistically significant.

3.2. Genetic Predictors of Poor Prognosis. Crude and adjusted odds ratios for the association between studied SNPs and poor prognosis are shown in Table 2 . In univariate analysis including all disease categories we found significant associations with poor prognosis for allele A of TNFA1P3/20 rs6920220, allele G of TRAF1/C5 rs3761847, and allele G of PTPN2 rs7234029. In multivariate models adjusted for relevant clinical predictors (disease category, DMARD treatment, and physician VAS) the association for TRAF1/C5 rs3761847 (1.96 [1.17-3.30]) remained significant at the 5\% level while TNFA1P3/20 rs6920220 (1.67 [0.98-2.83]) and PTPN2 rs7234029 (1.75 [0.99-3.10]) were no longer significant.

In the univariate analysis for the polyarticular categories we found associations for allele A of CTLA4 rs3087243 and allele G of PTPN2 rs7234029. After adjusting for clinical factors the associations for CTLA4 rs3087243 (2.90 [1.396.08]) and PTPN2 rs7234029 (3.30 [1.48-7.37]) were still significant at the $5 \%$ level.

Nevertheless, none of associations found was significant after the Bonferroni correction $(P<0.0016)$.

\section{Discussion}

The aim of our study was to identify genetic and clinical predictors of poor outcome in JIA. In a Portuguese sample of patients with JIA, we have not found genetic associations with a poor outcome. Longer duration of DMARD treatment, higher physician VAS, and polyarticular categories of JIA had a significant association with poor prognosis.

A growing number of studies have been focused on susceptibility to JIA, including genome wide association studies [21]. However, studies on genetics of JIA outcomes are still scarce. In a recent systematic literature review of early predictors of prognosis in JIA [8], the authors concluded that demographic, clinical, and laboratory values were insufficient to predict the individual prognosis. The authors also pointed out that hardly any other potential predictors were evaluated, such as cytokine levels, cell characteristics, results of imaging obtained early in the disease course, or genetic evaluations, such as HLA and SNPs in genes with a known function in the immune system.

There are some examples of genetic research on JIA outcomes, including a study that suggests that the MIF-173 polymorphism $(M I F-173 * \mathrm{C}$ allele $)$ is a predictor of poor outcome in systemic-onset JIA [22], another study that found SNPs in the IL6 gene associated with pain [14] and a correlation between TGF-b1 gene codon 25 genotypes and early radiological damage [14], and, in the ERA subtype, a publication suggesting that the presence of $H L A-D R B 1 * 08$ predicts failure to attain disease remission [23].

RA shares several clinical and pathological features with JIA and previous studies reported considerable overlap in genetic susceptibility loci for the two diseases [24-26]. JIA is a heterogeneous disease and genetic differences across the JIA categories and some category-specific effects have been identified [27, 28]. However, stratified analysis leads to small sample sizes for many of the categories. Larger cohorts of the ILAR categories are required to improve the power to detect any category-specific effects. We have stratified our analysis to investigate the polyarticular categories (polyarticular RF positive, polyarticular RF negative, and extended oligoarticular) which are the largest category in our sample.

We have found an association between a variant in the TRAF1/C5 locus and poor prognosis in Portuguese with JIA regardless of the disease category. Only in the polyarticular category of JIA did we find an association between 2 variants in the CTLA4 and PTPN2 loci and a poor outcome. Nevertheless, none of the associations found was significant after the Bonferroni correction was applied $(P<0.0016)$.

The analysis of the clinical variables identified a number of parameters associated with poor outcome. Patients with a poor prognosis were more likely to have polyarticular categories of JIA (polyarticular RF negative, polyarticular RF positive, and extended oligoarticular), to be on treatment with DMARDs for a longer period, and to have higher values of physician VAS at the last visit. Additionally, patients with a poor prognosis were less likely to have persistent oligoarticular JIA. Our results are in accordance with other studies that revealed that children with persistent oligoarticular JIA have a substantially better outcome than those with either systemic or polyarticular JIA, as measured by attaining remission, degree of disability, and structural damage $[8,10,11]$.

There are some limitations in our study, namely, the definition used to determine poor prognosis. There is no universal definition of "poor prognosis" in patients with JIA. We have chosen to integrate in our definition of "poor 
prognosis" two variables: (1) an instrument that combines disease activity and damage (CHAQ/HAQ); (2) the need for biological treatment, because patients that do not respond to conventional DMARDs, namely, methotrexate, have a higher chance of a poor outcome. Regarding this last point, our study included patients at different phases of their disease and we are aware that the access to biological therapy could not have been the same for all patients, leading to a selection bias. In addition, some patients could have started biological therapy mainly for extra-articular manifestations of the disease (e.g., uveitis) and not due to joint disease. This could also have potentially confounded our results.

Another limitation of our study is the problem of multiple comparisons: our results may simply be attributable to chance. The sample size in our cohort was too small to adequately test replication and a further study in a larger cohort is still required in order to confirm or refute our findings.

\section{Conclusions}

In summary, our study does not confirm the association between a panel of selected SNPs and poor prognosis in Portuguese patients with JIA. A search for additional genetic variants is required. Moreover, combination of genetic factors together with environmental exposures should also be considered. Further studies, in different populations of JIA patients, should be performed to replicate these findings.

\section{Abbreviations}

JIA: Juvenile idiopathic arthritis

DMARD: Disease Modifying Antirheumatic Drug

CHAQ: Childhood Health Assessment Questionnaire

SNP: $\quad$ Single nucleotide polymorphism

VAS: Visual analogue scale

MAF: $\quad$ Minor allele frequency

OR: $\quad$ Odds ratio

RF: $\quad$ Rheumatoid factor

ERA: Enthesitis-related arthritis.

\section{Disclosure}

There are no financial relationships relevant to the work.

\section{Conflict of Interests}

The authors have declared no conflict of interests.

\section{Acknowledgments}

This study would not have been possible without the collaboration of numerous clinicians, patients, and their parents. Funding for the genetic analysis was provided by an unrestricted grant from Abbvie, USA.

\section{References}

[1] A. Ravelli and A. Martini, "Juvenile idiopathic arthritis," The Lancet, vol. 369, no. 9563, pp. 767-778, 2007.
[2] L. N. Moorthy, M. G. E. Peterson, A. L. Hassett, and T. J. A. Lehman, "Burden of childhood-onset arthritis," Pediatric Rheumatology, vol. 8, article 20, 2010.

[3] A. Ravelli and A. Martini, "Early predictors of outcome in juvenile idiopathic arthritis," Clinical and Experimental Rheumatology, vol. 21, no. 5, pp. S89-S93, 2003.

[4] K. Oen, "Long-term outcomes and predictors of outcomes for patients with juvenile idiopathic arthritis," Best Practice and Research: Clinical Rheumatology, vol. 16, no. 3, pp. 347-360, 2002.

[5] B. Flatø, G. Lien, A. Smerdel et al., "Prognostic factors in juvenile rheumatoid arthritis: a case-control study revealing early predictors and outcome after 14.9 years," Journal of Rheumatology, vol. 30, no. 2, pp. 386-393, 2003.

[6] G. Z. Susic, R. M. Stojanovic, N. N. Pejnovic et al., "Analysis of disease activity, functional disability and articular damage in patients with juvenile idiopathic arthritis: a prospective outcome study," Clinical and Experimental Rheumatology, vol. 29, no. 2, pp. 337-344, 2011.

[7] M. Bartoli, M. Tarò, S. Magni-Manzoni et al., “The magnitude of early response to methotrexate therapy predicts long-term outcome of patients with juvenile idiopathic arthritis," Annals of the Rheumatic Diseases, vol. 67, no. 3, pp. 370-374, 2008.

[8] E. H. van Dijkhuizen and N. M. Wulffraat, "Early predictors of prognosis in juvenile idiopathic arthritis: a systematic literature review," Annals of the Rheumatic Diseases, 2014.

[9] K. Oen, "Long-term outcomes and predictors of outcomes for patients with juvenile idiopathic arthritis," Best Practice and Research: Clinical Rheumatology, vol. 16, no. 3, pp. 347-360, 2002.

[10] N. Adib, A. Silman, and W. Thomson, "Outcome following onset of juvenile idiopathic inflammatory arthritis: I. Frequency of different outcomes," Rheumatology, vol. 44, no. 8, pp. 995-1001, 2005.

[11] N. Adib, A. Silman, and W. Thomson, "Outcome following onset of juvenile idiopathic inflammatory arthritis: II. Predictors of outcome in juvenile arthritis," Rheumatology, vol. 44, no. 8, pp. 1002-1007, 2005.

[12] R. E. Petty, T. R. Southwood, P. Manners et al., "International League of Associations for Rheumatology classification of juvenile idiopathic arthritis: second revision, Edmonton, 2001," Journal of Rheumatology, vol. 31, no. 2, pp. 390-392, 2004.

[13] J. A. Melo-Gomes, N. Ruperto, H. Canhao et al., "The Portuguese version of the Childhood Health Assessment Questionnaire (CHAQ) and the Child Health Questionnaire (CHQ)," Clinical and Experimental Rheumatology, vol. 19, no. 4, supplement 23, pp. S126-S130, 2001.

[14] K. Oen, P. N. Malleson, D. A. Cabral et al., "Cytokine genotypes correlate with pain and radiologically defined joint damage in patients with juvenile rheumatoid arthritis," Rheumatology, vol. 44, no. 9, pp. 1115-1121, 2005.

[15] K. L. Hyrich, S. D. Lal, H. E. Foster et al., "Disease activity and disability in children with juvenile idiopathic arthritis one year following presentation to paediatric rheumatology. Results from the childhood arthritis prospective study," Rheumatology, vol. 49, no. 1, pp. 116-122, 2010.

[16] K. Oen, P. N. Malleson, D. A. Cabral et al., "Early predictors of longterm outcome in patients with juvenile rheumatoid arthritis: subset-specific correlations," Journal of Rheumatology, vol. 30, no. 3, pp. 585-593, 2003.

[17] N. Ruperto, A. Ravelli, J. E. Levinson et al., "Longterm health outcomes and quality of life in American and Italian inception 
cohorts of patients with juvenile rheumatoid arthritis. II. Early predictors of outcome," Journal of Rheumatology, vol. 24, no. 5, pp. 952-958, 1997.

[18] M. Vivarelli, L. E. D’Urbano, A. Insalaco et al., "Macrophage migration inhibitory factor (MIF) and oligoarticular juvenile idiopathic arthitis (o-JIA); association of MIF promoter polymorphisms with response to intra-articular glucocorticoids," Clinical and Experimental Rheumatology, vol. 25, no. 5, pp. 775781, 2007.

[19] A. Berdeli, A. R. Özyürek, Z. Ülger et al., "Association of macrophage migration inhibitory factor gene $-173 \mathrm{G} / \mathrm{C}$ polymorphism with prognosis in turkish children with juvenile rheumatoid arthritis," Rheumatology International, vol. 26, no. 8, pp. 726-731, 2006.

[20] R Core Team, R: A Language and Environment for Statistical Computing, R Foundation for Statistical Computing, Vienna, Austria, 2013, http://www.R-project.org/.

[21] A. Hinks, J. Cobb, M. C. Marion et al., "Dense genotyping of immune-related disease regions identifies 14 new susceptibility loci for juvenile idiopathic arthritis," Nature Genetics, vol. 45, no. 6, pp. 664-669, 2013.

[22] F. de Benedetti, C. Meazza, M. Vivarelli et al., "Functional and prognostic relevance of the -173 polymorphism of the macrophage migration inhibitory factor gene in systemic-onset juvenile idiopathic arthritis," Arthritis and Rheumatism, vol. 48, no. 5, pp. 1398-1407, 2003.

[23] B. Flatø, A.-M. Hoffmann-Vold, A. Reiff, Ø. Førre, G. Lien, and O. Vinje, "Long-term outcome and prognostic factors in enthesitis-related arthritis: a case-control study," Arthritis \& Rheumatism, vol. 54, no. 11, pp. 3573-3582, 2006.

[24] A. Hinks, J. Cobb, M. Sudman et al., "Investigation of rheumatoid arthritis susceptibility loci in juvenile idiopathic arthritis confirms high degree of overlap," Annals of the Rheumatic Diseases, vol. 71, no. 7, pp. 1117-1121, 2012.

[25] S. D. Thompson, M. Sudman, P. S. Ramos et al., "The susceptibility loci juvenile idiopathic arthritis shares with other autoimmune diseases extend to PTPN2, COG6, and ANGPT1," Arthritis and Rheumatism, vol. 62, no. 11, pp. 3265-3276, 2010.

[26] H. M. Albers, F. A. S. Kurreeman, J. J. Houwing-Duistermaat et al., "The TRAF1/C5 region is a risk factor for polyarthritis in juvenile idiopathic arthritis," Annals of the Rheumatic Diseases, vol. 67, no. 11, pp. 1578-1580, 2008.

[27] A. Hinks, P. Martin, E. Flynn et al., "Subtype specific genetic associations for juvenile idiopathic arthritis: ERAP1 with the enthesitis related arthritis subtype and IL23R with juvenile psoriatic arthritis," Arthritis Research and Therapy, vol. 13, no. 1, article R12, 2011.

[28] C. J. W. Stock, E. M. Ogilvie, J. M. Samuel, M. Fife, C. M. Lewis, and P. Woo, "Comprehensive association study of genetic variants in the IL-1 gene family in systemic juvenile idiopathic arthritis," Genes and Immunity, vol. 9, no. 4, pp. 349-357, 2008. 


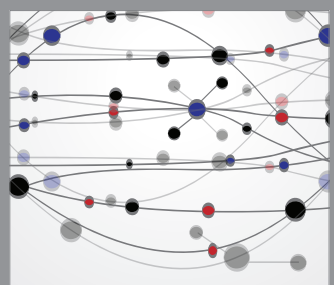

The Scientific World Journal
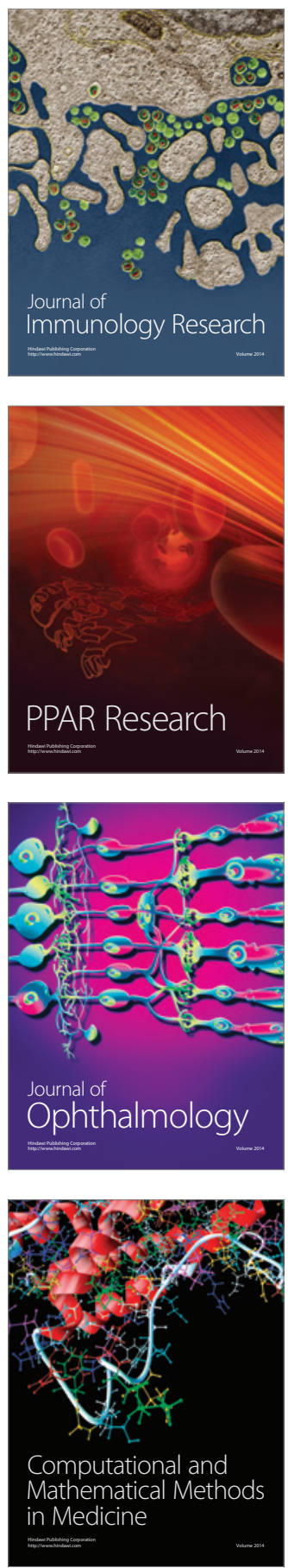

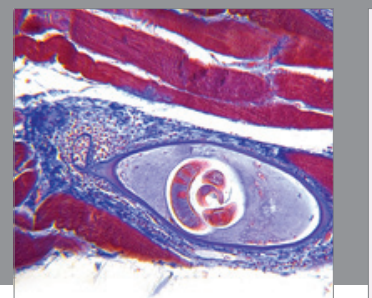

Gastroenterology

Research and Practice
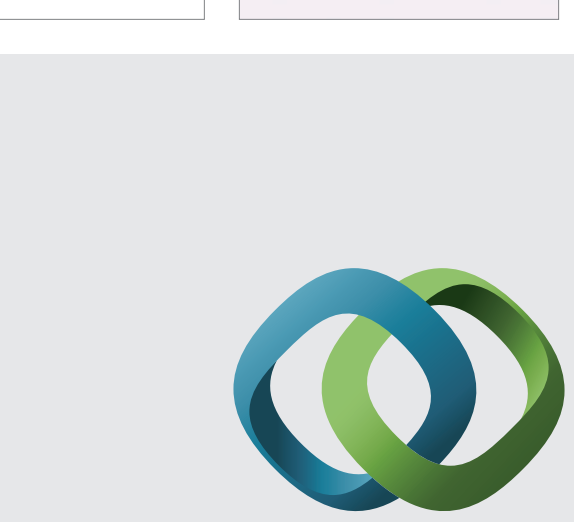

\section{Hindawi}

Submit your manuscripts at

http://www.hindawi.com
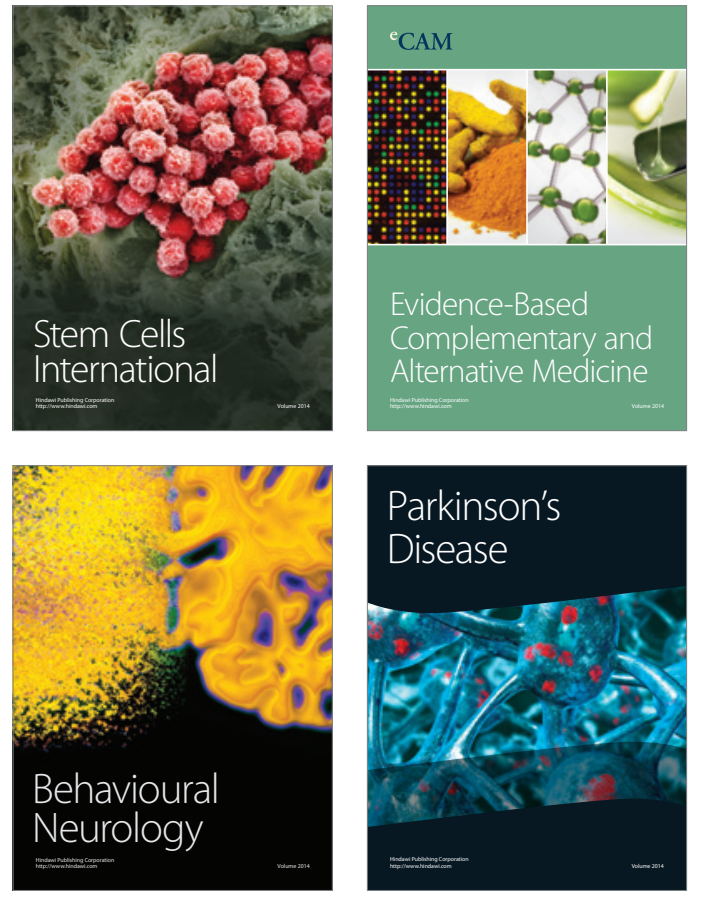
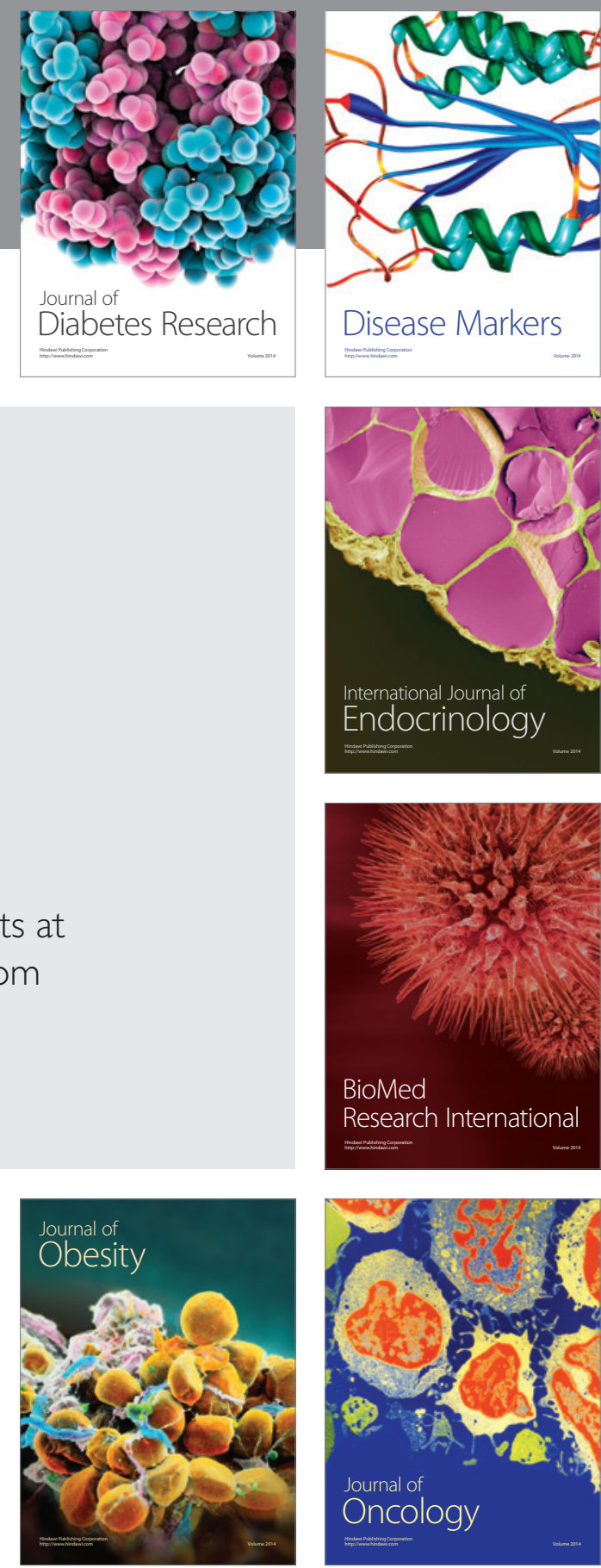

Disease Markers
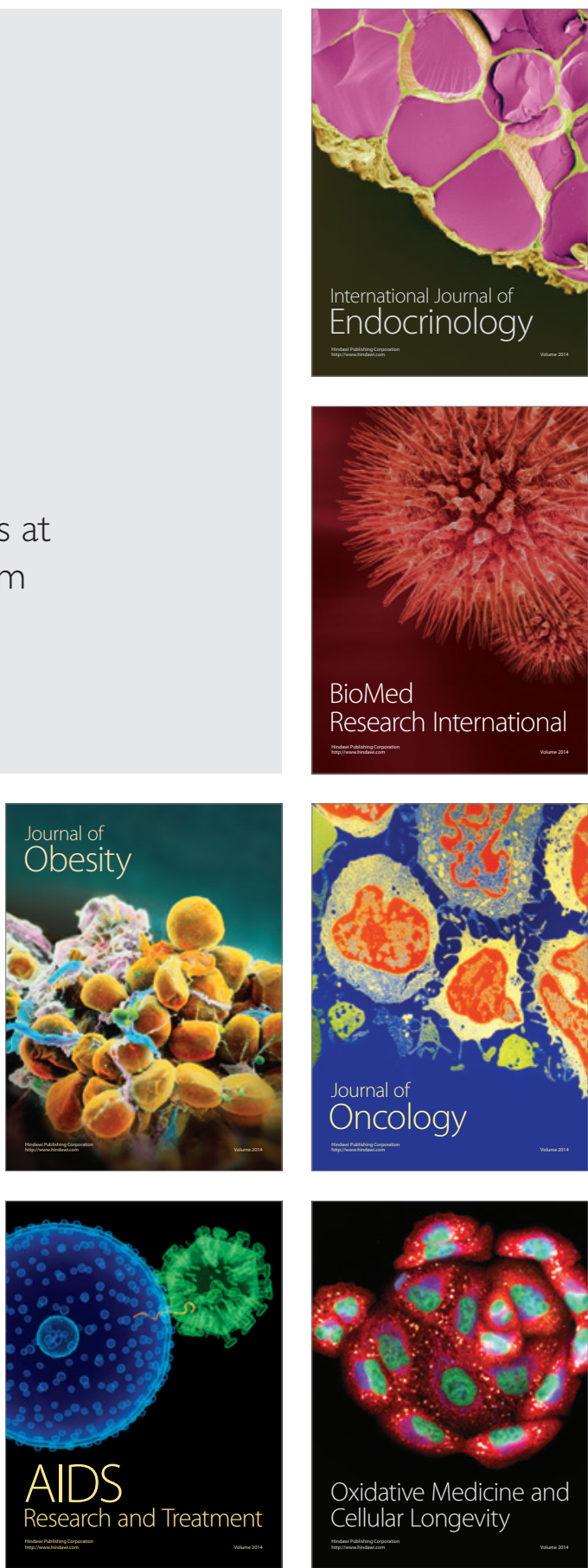